\title{
Bridging the gap between theory and practice in LPV fault detection for flight control actuators is
}

\author{
Bálint Vanek*, András Edelmayer, Zoltán Szabó, József Bokor \\ Computer and Automation Research Institute, Hungarian Academy of Sciences, Kende utca 13.-17., Budapest H-1111, Hungary
}

\section{A R T I C L E I N F O}

Article history:

Received 30 April 2013

Accepted 2 May 2014

Keywords:

Fault detection and isolation (FDI)

Linear parameter-varying (LPV) systems

Robust estimation

Commercial aircraft

Analytical redundancy

\begin{abstract}
A B S T R A C T
Two different approaches for fault detection, the geometric and the detection filter based methods, are compared in the paper from practical aspects, using the linear parameter-varying (LPV) framework. Presenting two designs allows a comparison of global, system level, and local component level fault detection methods with special emphasis on their relevance to aircraft industry. Practical engineering design decisions are highlighted via applying them to a high-fidelity commercial aircraft problem. The successive steps of the design, including fault modeling, LPV model generation, and LPV FDI filter synthesis, including implementation aspects, are discussed. Results are presented according to the industrial assessment perspectives phrased within the EU ADDSAFE project.
\end{abstract}

(c) 2014 Elsevier Ltd. All rights reserved.

\section{Introduction}

Modern fly-by-wire aircraft flight control systems are becoming more complex with many actuators controlling several aerodynamic surfaces. While performance goals, including aerodynamic drag minimization and structural design optimization, are becoming more and more important, flight must be kept at the same highest safety level. In parallel, there is a clear trend towards the MoreElectric Aircraft. Recently, Airbus introduced on the A380 a new hydraulics layout (Van den Bossche, 2006), where the three hydraulics circuitry is replaced by a two hydraulics plus two electric layout, which saves significant mass for the aircraft. Each primary surface has a single hydraulically powered actuator and electrically powered back-up with the exception of the outer aileron, which uses the two hydraulic systems together. Consequently, the trends of complexity and more-electric architectures, like electromechanical actuators (EMA) with more fault sources, raise the importance of availability, reliability and operating safety, while all aircraft manufacturers must be compliant with stringent safety regulations of FAA, EASA and other aviation authorities. The newer societal imperatives towards an environmentally friendlier aircraft, with still the highest level of safety and reliability, can only be achieved with advanced on-line supervision and fault diagnosis methods relying on analytical redundancy. The traditional approach to fault diagnosis in the wider application context is based on hardware redundancy methods which use multiple sensors, actuators computers and software to

This work was funded by the EU FP7 program ADDSAFE, Contract no. FP7-AAT-2008-233815, Andres Marcos program manager

* Corresponding author. Tel.: +361279 6000; fax: +3614667483.

E-mail address: vanek@sztaki.hu (B. Vanek). measure and control a particular variable (Goupil, 2011). Based on the mathematical model of the plant, analytical relation between different sensor outputs can be used to generate diagnostics signals, often called residuals. There is a growing interest in methods which do not require additional hardware redundancy, and only rely on the ever increasing level of computational power onboard the aircraft.

In analytical redundancy schemes, the resulting difference generated from the consistency checking of different variables is called residual signal. The residual should be zero when the system is normal, and should diverge from zero when a fault occurs in the system. This zero and non-zero property of the residual is used to determine whether or not faults have occurred. Analytical redundancy makes use of a quantitative mathematical model of the system, and the goal is the determination of faults from the comparison of available system measurements with a priori information represented by the mathematical model, through generation of residual quantities and their analysis. In parallel with the residual generation the analytical redundancy within the systems can be used to generate virtual sensors, which can complement the set of physically redundant sensors. Various approaches have been applied to the residual generation problem, the parity space approach (Chow \& Willsky, 1984), the multiple model method (Chang \& Athans, 1978), detection filter design using a geometric approach (Massoumnia, 1986), frequency domain concepts (Frank, 1990), unknown input observer concept (Chen \& Patton, 1999), dynamic inversion based detection (Edelmayer, Bokor, \& Szabo, 2003), sliding mode observers (Alwi, Edwards, \& Marcos, 2010), extended Kalman filter based parameter estimation (Eykeren, Chu, \& Mulder, 2012) and using rational nullspace bases (Varga, 2003). Most of these design approaches refer to linear time-invariant (LTI) systems. The geometric concept is further generalized to linear 
parameter-varying (LPV) systems by Balas, Bokor, and Szabo (2003), while input affine nonlinear systems are considered by De Persis, De Santis, and Isidori (2001). The basic concepts underlying observer-based fault detection and isolation (FDI) schemes are the generation of residuals and the use of an optimal or adaptive threshold function to differentiate faults from disturbances, as surveyed in Frank (1990) and Patton and Chen (1996). The threshold function is used to robustify the detection of the fault by minimizing the possibility of false alarms and missed detections. The effect of disturbances and model uncertainty have to be minimized on the residuals. For fault isolation, the generated residual has to include enough information to differentiate said fault from another, which is accomplished through structured residuals or directional vectors. Robustness of the FDI algorithm is determined by its capability to decouple the filter performance outputs from disturbances, errors, and unmodelled dynamics.

Advanced design methods relying on the robust control machinery tend to be very complex and difficult to implement in practical engineering systems, hence it is important to show the applicability of different methods and their corresponding computational complexity relative to the requirements of an aircraft manufacturer. The importance of this paper is on the comparison of the global (aircraft level) and local (component level) methods with respect to their detection performance, development complexity and implementation aspects. An aileron fault detection case is handled by a geometric FDI filter (Vanek, Szabó, Edelmayer, \& Bokor, 2011) using an aircraft level mathematical model, while the elevator fault detection is tackled by a more conventional detection filter based approach (Vanek, Szabó, Edelmayer, \& Bokor, 2012) using the local mathematical description of the hydraulic actuator.

The remainder of the paper is structured as follows. Section 2 presents the basic concepts of geometric and detection filter based fault detection filter design. The motivating example, a civil aircraft, is described in Section 3. The methods are applied to the high fidelity aircraft example, which demonstrates the proposed approach, given in Section 4. The lessons learnt during coding the algorithms in hardware ready implementation are discussed in Section 5. Finally, the paper is concluded in Section 6 .

\section{LPV FDI}

There are a number of analytical redundancy based FDI methods available in the literature for linear and nonlinear systems. While recent nonlinear approaches are useful for the analysis, and partly for the design of detection filters, they are largely incapable of solving synthesis problems due to the computational burden they usually pose for the implementation. LPV modeling is known to be a capable approach to alleviate this problem; it has been useful in many areas of control and filtering in handling nonlinear problems. The idea suggests that a broad class of nonlinear system models can be converted into a quasi-linear form (Tan, 1997), obtaining the socalled quasi-linear parameter-varying (qLPV) representation. The state-space matrices depend affinely on a parameter vector in $\mathrm{QLPV}$ systems. This approach is particularly appealing when the nonlinear plant can be considered as linear one assuming the presence of a set of time varying scheduling parameters in the system matrices. The parameters are thought not necessarily known at the design stage but always measurable real-time. This class of systems can be described as

$$
\begin{aligned}
& \dot{x}(t)=A(\rho) x(t)+B(\rho) u(t)+\sum_{j=1}^{m} L_{j}(\rho) f_{j}(t) \\
& y(t)=C(\rho) x(t)+D(\rho) u(t)+\sum_{j=1}^{m} M_{j}(\rho) f_{j}(t)
\end{aligned}
$$

where $x(t)$ is the state vector, $u(t)$ is the input vector and $y(t)$ is the output vector of the system, while there are $j$ different failure signals $f_{j}$ affecting the system, the $A(\rho), B(\rho), C(\rho), D(\rho)$ matrices are parameter dependent. $L_{j}$ are the directions of the faults acting on the input, most often on the actuators, while $M_{j}$ are the output fault directions most often acting on the sensors. In a particular FDI filter synthesis problem the goal is to detect a subset of these faults and being insensitive to the rest of them.

\subsection{Geometric LPV FDI}

The geometric design approach (Bokor \& Balas, 2004; Massoumnia, 1986) is known for its excellent fault isolation, fault reconstruction and sensitivity properties under small modeling uncertainty and noise. Vanek, Szabo et al. (2011) show the application of the geometric LPV FDI approach to a complex, 6 degreesof-freedom (DOF) rigid body aircraft model. Previous approaches (Khong \& Shin, 2007; Szaszi, Marcos, Balas, \& Bokor, 2005) only considered the longitudinal dynamics of the airplane, which is applicable for the elevator fault detection case but as described in Vanek, Seiler, Bokor, and Balas (2011) designs based on decoupled dynamics are inherently limited to detection around the trim (cruise) flight condition and might be less robust to deviation from the trim operating point.

The derivation of the geometric FDI filter solving the fundamental problem of residual generation (FPRG) is briefly presented for the LTI case (Massoumnia, 1986) with no disturbance, no uncertainty and the detection and isolation of two faults, for illustration purposes. The LPV synthesis can be found in Balas et al. (2003).

Consider the LTI system with two additive actuator faults:

$\dot{x}(t)=A x(t)+B u(t)+L_{1} f_{1}(t)+L_{2} f_{2}(t)$

$y(t)=C x(t)$

where $L_{1}$ and $L_{2}$ represent the fault directions in the state space. $f_{1}$ and $f_{2}$ are the fault signals. The fault signals are zero if there is no fault but nonzero if the particular fault occurs. Only actuator faults are considered here but sensor faults can also be considered within the theory. The FPRG can be phrased as synthesizing residual generators (filters) with outputs $r_{i}(i=1,2)$ that have the following decoupling property: $r_{i}$ is sensitive to $f_{i}$ but insensitive to $f_{j}, i \neq j$. More precisely, if $f_{i}=0$ then $\lim _{t \rightarrow \infty} r_{i}(t)=0$ and if $f_{i} \neq 0$ then $r_{i} \neq 0$.

The solution of this problem depends on the $(C, A)$-invariant subspaces and certain unobservability subspaces (Massoumnia, 1986). A ( $C, A)$-unobservability subspace $\mathcal{S}$ is a subspace such that there exist matrices $G$ and $H$ with the property that $\mathcal{S}$ is the maximal $(A+G C)$ invariant subspace contained in Ker $H C$. The family of $(C, A)$-unobservability subspaces containing a given set $\mathcal{L}$ has a minimal element. Define $\mathcal{L}_{i}=\operatorname{Im} L_{i}(i=1,2)$ and denote by $\mathcal{S}^{*}$ the smallest unobservability subspace containing $\mathcal{L}_{2}$. Then the fundamental problem of residual generation has a solution if and only if $\mathcal{S}^{*} \cap \mathcal{L}_{1}=0$ (Massoumnia, Verghese, \& Willsky, 1989). The condition $\mathcal{S}^{*} \cap \mathcal{L}_{1}=0$ ensures that the fault to be detected is not hidden in the unobservability subspace of the detection filter. In fact, the fault direction will be decoupled from the rest of the fault directions since they are contained in the unobservability subspace of the residual generator. This result can be extended to LPV systems (Balas et al., 2003) and to nonlinear input affine systems (De Persis et al., 2001).

The residual generator associated with fault direction $L_{1}$ can be described by an observer of the following form:

$\dot{w}(t)=N w(t)-G y(t)+F u(t)$

$r_{1}(t)=M w(t)-H y(t)$

where $u$ and $y$ are the known input and measured output signals of the original LTI system. $w$ is the state vector of the residual 
generator and $r_{1}$ is the residual. The state space matrices can be determined as follows (Massoumnia, 1986). $H$ is a solution of the equation $\operatorname{Ker} H C=\operatorname{Ker} C+\mathcal{S}^{*}$, and $M$ is the unique solution of $M P=H C$, where $P$ is a projection operator mapping the entire state space into a factor space with special properties $P: \mathcal{X} \rightarrow \mathcal{X} / \mathcal{S}^{*}$. Consider a gain matrix $\hat{G}$ chosen such that $(A+\hat{G} C) \mathcal{S}^{*} \subseteq \mathcal{S}^{*}$ and define $\hat{A}=P(A+G C) P^{T}$. $\hat{A}$ is not necessarily Hurwitz. To obtain quadratically stable filters one can set $N=\hat{A}+\tilde{G} M$, where $\tilde{G}:=X^{-1} K$ and $X, K$ are determined from the linear matrix inequality (LMI):

$0 \geqslant \hat{A}^{T} X+X \hat{A}+M^{T} K^{T}+K M$

$0 \bar{X}=X^{T}$

Then set $G=P \hat{G}+\tilde{G} H$ and $F=P B$. Using this approach there are as many filters as faults to detect, and their state dimensions are equal to the consecutive dimensions of the factor spaces $\mathcal{X} / \mathcal{S}^{*}$. The filter poles can be tuned by suitable output injection, which is formulated as imposing constraints in the LMIs above, resulting in perfect reconstruction of fault signals $f_{i}$. One issue is that the filter design does not consider model uncertainty and the fault detection performance may not be robust. It is a standard procedure to pose the FDI filter design problem as an $\mathcal{H}_{\infty}$ optimization (Marcos, Ganguli, \& Balas, 2005), which uses robust control methods. In the one-step robust $\mathcal{H}_{\infty}$ optimization approach fault reconstruction is achieved as a model matching problem in one step, without exact decoupling properties, and approximate disturbance decoupling is achieved in the $\mathcal{H}_{\infty}$ optimal sense. On the other hand the geometric (FPRG) approach is able to provide exact decoupling between the fault(s) and the disturbances, but the resulting FPRG filter dynamics might not be optimal for the detection purpose. To overcome this weakness of the geometric approach, the FPRG filter can be augmented with a post-filter (Vanek, Szabo et al., 2011), as shown in Fig. 1, where the dynamics of the FPRG filter is shaped with suitable output injection $o$. Fault reconstruction is achieved with model matching using the $\mathcal{H}_{\infty}$ optimal filter $F D I_{h 1}$, which uses the independent outputs of the FPRG filter $r_{f}$, and generates a residual $r_{s}$ which tracks the ideal fault response $f_{i d}$. The main advantage of this approach is that elements of $r_{f}$ are all decoupled from the disturbances and hence the filter $F D I_{h} 1$ only acts on the signals which are sensitive to the faults and can be fed back to shape the dynamics of the FPRG filter, moreover it is often not feasible to solve the LPV $\mathcal{H}_{\infty}$ problem for large plants, but the geometric LPV FPRG filter requires only algebraic computations and no optimization is involved. It is shown in Seiler, Vanek, Bokor, and Balas (2011) that the design has an interesting self-optimality property for input multiplicative uncertainty sets. Specifically, the filter designed on the nominal plant is the optimal filter in the robust model matching problem assuming input multiplicative uncertainty.

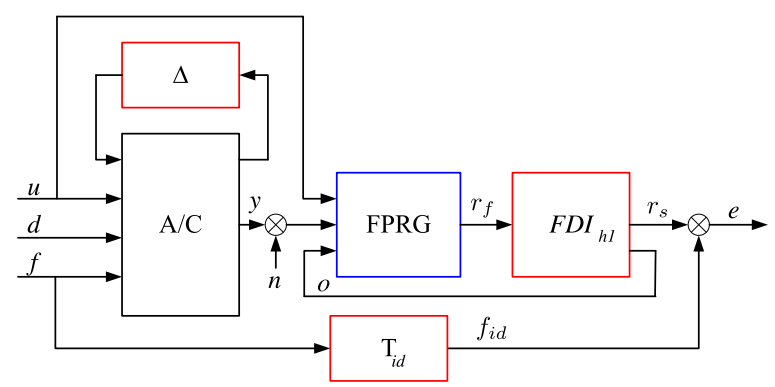

Fig. 1. Geometric FPRG filter with $\mathcal{H}_{\infty}$ augmentation in the model matching framework.

\subsection{Detection filter based LPV FDI}

The basic idea behind the LTI observer based approaches is to estimate the outputs of the system from the measurements by using a Luenberger observer, assuming a deterministic setting or a Kalman filter in the stochastic case. Then the weighted output estimation error is used as a residual. The flexibility in selecting observer gains and designing static or time varying thresholding functions is fully exploited in the literature (Frank, Ding, \& Koppen-Seliger, 2000). The interest is in LPV FDI, which is a generalization of the LTI case, where the goal is the estimation of the outputs using an observer, whilst the estimation of the state vector is unnecessary. As a matter of fact, a functional observer is suitable for this task. In practice, the order of the functional observer is less than the order of the state observer. It is desired to estimate the output, a linear (parameter-varying) function of the state, i.e. $C(\rho) x(t)$, using a functional or generalized LPV Luenberger-like observer with the following structure:

$\dot{z}(t)=F(\rho) z(t)+K(\rho) y(t)+J(\rho) u(t)$

$w(t)=G(\rho) z(t)+R(\rho) y(t)+S(\rho) u(t)$

$\hat{y}(t)=w(t)+D(\rho) u(t)$

$r(t)=Q[y(t)-\hat{y}(t)]=Q_{1}(\rho) z(t)+Q_{2}(\rho) y(t)+Q_{3}(\rho) u(t)$

where $z(t) \in \mathcal{R}^{q}$ is the state vector of the functional observer, $F, K, J$, $R, G$ and $S$ are matrices with appropriate dimensions. The output $w$ $(t)$ of this observer is said to be an estimate of $C(\rho) x(t)$, the output of the system without the feedthrough term, for the system in Eq. (1), in an asymptotic sense in the absence of faults. The residual $r(t)$ is generated based on the states of the observer, where the $Q_{i}$ entries are free parameters, but have to satisfy the following set of equations (Chen \& Patton, 1999):

$\operatorname{eig}(F(\rho))<0$

$T A(\rho)-F(\rho) T=K(\rho) C$

$J(\rho)=T B(\rho)-K(\rho) D$

$Q_{1}(\rho) T+Q_{2}(\rho) C=0$

$Q_{3}(\rho)+Q_{2}(\rho) D=0$

where $T$ is a coordinate transformation matrix, which can be constant if $C$ and $D$ in Eq. (1) are also constant. It can be seen that the residual depends solely on faults in the asymptotic sense, given a stable estimator dynamics.

\section{Mathematical model of the aircraft}

A global aircraft level mathematical model is derived for the aileron fault detection problem, since only a single measurement of surface deflection is available for FDI purposes. On the other hand, in the elevator fault detection problem a local model is able to provide the required analytical redundancy, since three independent measurements can be used for residual generation.

\subsection{General aircraft characteristics}

The aircraft model used in this paper is a generic civil aircraft from Airbus. A high-fidelity aerodynamic database, propriety of Airbus Operations S.A.S, is used within the project, but results in the present paper are normalised due to confidentiality reasons. The aircraft has two engines and a nominal weight of 200 tons. Some of its performance at cruise flight condition are speed of 240 knots, altitude of $30000 \mathrm{ft}$. The aircraft has 19 control inputs, 
and measurement of 6-DOF motion with load factor $\left(n_{x}, n_{y}, n_{z}\right)$, body rate $(p, q, r)$, velocity $\left(V_{T}\right)$, aerodynamic angles $(\alpha, \beta)$, position $(X, Y, Z)$ and attitude $(\phi, \theta, \psi)$ outputs. The inputs are standard left and right engine throttle; airbrake (which is disabled at cruise flight condition), left and right internal and external ailerons, six spoilers on each side, left and right elevators, rudder and trimmable horizontal stabilizer which is used mainly for trimming purposes. The nonlinear body-axes rigid body dynamics includes 12 states: $p, q, r$ body rates, $u, v, w$ velocities all in body axes, $\phi, \theta, \psi$ Euler angles, representing the rotation between the body and inertial axes, and $X, Y, Z$ positions in the north-east-down coordinate frame, assuming flat Earth for simplicity. The rigid body aircraft model is augmented with nonlinear actuator and sensor models on all input and output channels (Goupil, 2011).

\subsection{Deriving a reliable LPV model of the aircraft}

The ADDSAFE benchmark aircraft model is given in a nonlinear simulation, where several components are given as black-box models. Aerodynamic coefficients are represented by trained neural-networks, for which it is not possible to derive affine LPV models in the form of Eq. (2) analytically. Based on the required operating envelope it is decided to have weight $(\mathrm{m})$, center of gravity position (c.g.), altitude above sea level $(h)$, and calibrated airspeed $\left(V_{\text {cas }}\right)$ as scheduling variables. The nonlinear model is then trimmed and linearized at different points of the operating envelope to obtain pointwise LTI models.

The LTI models of the aircraft are obtained at level flight, with $p=q=r=0 \mathrm{rad} / \mathrm{s}, v_{x}=$ const. $\mathrm{m} / \mathrm{s}, v_{y}=0 \mathrm{~m} / \mathrm{s}, v_{z}=$ const. $\mathrm{m} / \mathrm{s}$, at various altitudes, see Vanek, Seiler et al. (2011) for details. The airbrake, which is disabled at high Mach numbers, is removed from the control inputs since it has no effect on the aircraft. The model used for trim is an open-loop model without the onboard controller implemented in the flight control computer (FCC), since actuators and sensors are assumed to have unit steady state gain and low-pass characteristics, their dynamics are omitted. Trim is obtained with zero aileron, rudder and elevator deflection, left and right engines are providing the same amount of thrust to balance the yawing motion. Pitch axis trim is obtained with the trimmable horizontal stabilizer, while the aircraft has a constant angle-ofattack.

The pointwise LTI systems have nine states, since the fault detection problem is invariant of $X, Y$ positions and $\psi$ heading angle. All systems are stable, which is necessary for estimator based FDI techniques. After investigation of the FCC commands, assuming faults appear only on the aileron, elevator, and rudder channels, the inputs of the system are simplified. The two engines are receiving the same commands, the spoilers have a fixed coupling, the two elevators are also moving in unison, hence the number of inputs can be reduced to 9, namely pi engine; $\delta_{a, L}$ Aileron internal left; $\delta_{a, I R}$ Aileron internal right; $\delta_{a, E L}$ Ail external left; $\delta_{a, E R}$ Ail external right; $\delta_{s p}$ Spoiler; $\delta_{e}$ Elevator; $\delta_{\mathrm{r}}$ Rudder; and $\delta_{\text {THS }}$ trimmable horizontal stabilizer. The resulting LTI models are augmented with first-order sensor and actuator dynamics derived from the high-fidelity simulation, to account for their effect on the aircraft behavior and also three directional wind disturbance is perturbing the model via Dryden wind gust filters. These LTI models are then approximated by an affine LPV model using the DLR proprietary LFR toolbox. The procedure is described in detail in Hecker (2006).

When obtaining the $L P V$ representation, several simplifying assumptions are made to reduce the problem size. The LPV model is an open-loop approximation, with first-order affine dependence on the four scheduling parameters, without the control loop in feedback, since the complexity of the Airbus proprietary control logic is beyond tractable size. This leads to a conservative system

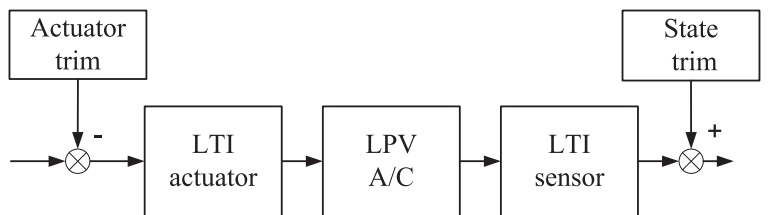

Fig. 2. Interconnection of the LPV rigid body equations with a sensor and actuator model.

description for FDI synthesis, since non-feasible system state trajectories have to be handled also. Moreover, trim input and output values are also computed at the operating points and interpolated linearly between them when simulating the LPV dynamics. When the nonlinear aircraft characteristics is compared to the behavior of the corresponding LPV A/C model the schematics used in Fig. 2 is used to account for the appropriate match.

The scenario under investigation is a left inboard aileron fault. To account for this fault, and to be able to consider additional faults as disturbances, the original affine LPV system description of the aircraft is augmented with additional fault directions as in Eq. (2):

$$
\begin{aligned}
\dot{x}(t)= & A(\rho(t)) x(t)+B(\rho(t)) u(t)+L_{1}(\rho(t)) f_{a, I L}(t) \\
& +L_{2}(\rho(t)) f_{e, L}(t)+L_{3}(\rho(t)) f_{r}(t)
\end{aligned}
$$

$\tilde{y}(t)=\tilde{C} x(t)$

where $L_{1}(\rho(t))=B(2,:)(\rho(t))$ is the corresponding column of the control effectiveness matrix of the left inboard aileron. $L_{2}(\rho(t))=$ $0.5 B(7,:)(\rho(t))$ corresponds to the left elevator control effectiveness, while $L_{3}(\rho(t))=B(8,:)(\rho(t))$ is the column of the rudder control effectiveness. It is important to note that although the interest is in detecting left internal aileron faults, the effect of additional actuator faults is also included. It is necessary to include them, since in the design stage the goal is not only to design an FDI filter which is sensitive to faults in the direction of $L_{1}$, but also to provide good isolation and to keep false alarm rate low, which is accomplished by the filter being insensitive to additional faults characterized by the directions of $L_{2}$ and $L_{3}$. On the other hand, adding more disturbance inputs to the augmented plant does not provide additional benefits, since the right aileron has exactly the same $B$ matrix as the left aileron with an opposite sign, hence these two faults are indistinguishable. The outer ailerons are creating a similar effect on the aircraft and are virtually identical to the inner ailerons when the measurements are corrupted by noise and system uncertainty. The left and the right elevator are merged into one input, but originally they have also the same column in their control effectiveness matrix. Although spoiler runaway can have a significant effect at high speed, the response is still smaller than a runaway of a primary control surface, hence spoilers are omitted from the investigation. Engines have significantly slower dynamics than the flight control surfaces and their diagnostics is well established within their full-authority digital engine control (FADEC) system, hence engines are also assumed fault free within this study.

It is also important to note that only a subset of sensor measurement outputs $(\tilde{y})$ are selected (by $\tilde{C}$ ) for the fault detection problem. Load factor has direct feedthrough from actuator inputs resulting in nonzero $D$ matrix, which is difficult to handle in the geometric FDI framework, even after filtering with actuator and sensor dynamics, hence these measurements are omitted.

The various fault scenarios to be investigated are disconnection, jamming and bias on the aileron actuator. In case of disconnection, the aileron surface goes to the null hinge moment position dictated by the aerodynamic forces acting on it, which is at a constant angle $\delta_{e}^{N H M}$. The failure signal is defined as $f_{a, i, L}(t)=\delta_{e}^{N H M}-u_{a, i, L}(t)$. The other scenario is liquid jamming, when a bias $b_{l, j}$ occurs on the actuator rod sensor, and hence on the rod position relative to 
the commanded deflection. This can be captured by a fault signal of $f_{a, i, L}(t)=b_{l, j}$. The third case is called solid jamming, when the actuator is stuck at a given position $b_{s, j}$ and does not move, this is similar to the first scenario, only the offset is not $\delta_{e}^{M I N}$ but some other quantity $b_{s, j}$.

\subsection{Nonlinear elevator actuator model}

The limited set of measurements on the aileron, only the actuator rod position is sent back to the FCC, makes it very difficult to apply the local model based FDI. On the other hand, in case of the elevator the 3 independent signals of servo valve position $\left(y_{s v}\right)$, rod position $\left(y_{\text {rod }}\right)$ and surface deflection $\left(y_{\text {surf }}\right)$ allow more analytic redundancy, suitable for local model based methods (see Fig. 3). The actuator model is based on the physical equation used to estimate the actuator rod speed as a function of the hydraulic pressure delivered to the actuator, and is a function of the external forces acting on the control surface and reacted by the actuator (Andrieu, 1999). The estimated position $\hat{p}$ results from a discrete integration (trapezoidal method) of this estimated velocity $(\hat{v})$. The two main contributors are the aerodynamic force (which depends also on $\hat{p}$ ) and the servo-control load in a damping mode of the adjacent passive actuator (actuators are installed in dual configuration to most of the flight control surfaces, see Goupil, 2010 for more details). The actuator rod speed is expressed as

$\hat{v}=\hat{v}_{c} \sqrt{\frac{\Delta P-\frac{F_{\text {aero }}+F_{\text {damping }}}{S}}{\Delta P_{\text {ref }}}}$

where $\Delta P$ is the hydraulic pressure really delivered to the actuator $F_{\text {aero }}$ represents the aerodynamic forces applied on the control surface. The corresponding model is not detailed here for confidentiality reasons, only a low-order representation of it is detailed without the servo valve dynamics, which is sufficient to capture its main behavior. $F_{\text {damping }}$ represents the servo-control load of the adjacent actuator in the damping mode, which always acts against the required motion $\left(v_{c}\right)$, hence always positive:

$F_{\text {damping }}=K_{d} \hat{v}^{2}$

$K_{d}$ is the actuator damping coefficient and $\hat{v}$ represents the speed to be estimated, $S$ is the actuator piston surface area, $\Delta P_{\text {ref }}$ is the differential pressure corresponding to the maximum rod speed. This speed is reached when the servo valve is fully opened, i.e. when $\Delta P=\Delta P_{\text {ref }} . \hat{v}_{c}$ is the rod speed computed by the flight control computer. It corresponds to the maximal speed of one actuator alone with no load.

The actuator rod position is obtained using the integration of the rod speed. In case of the elevator, the geometric deflection of the flight control surface is measured $\left(y_{\text {surf }}\right)$, which is directly related to the rod position via a static nonlinear relation, which is also measured $\left(y_{\text {rod }}\right)$, as shown in Fig. 3. While the servo valve position, used for the control of the hydraulic circuit, is also available for measurement $\left(y_{s v}\right)$.

\subsection{LPV aircraft actuator model}

As described above, the actuator servo-loop has position commands $\delta_{e}^{C M D}$, while measurement of three signals $\left(y_{\text {surf }}, y_{\text {rod }}\right.$, $\left.y_{s v}\right)$ are available, where the real measurements of $y_{\text {surf }}$ are associated with their model of $\delta_{e}$. The model structure is composed of two main blocks, as shown in Fig. 4, a static nonlinear mapping and a parameter-dependent dynamics leading to a Hammerstein-type representation. The aerodynamic forces $\left(F_{\text {aero }}\right)$ acting on the elevator surface are assumed to be represented by a static nonlinear mapping as a function of elevator deflection $\left(\delta_{e}\right)$, trimmable horizontal stabilizer deflection $\left(\delta_{T H S}\right)$, angle-of-attack $(\alpha)$, dynamic pressure $\left(P_{d}\right)$, and Mach number $(M)$. Based on the Airbus confidential high fidelity $F_{\text {aero }}$ model, a low-order polynomial representation is derived as a function of the variables:

$F_{\text {aero }}=C_{\delta}\left(\delta-C_{0}\right)+C_{\delta 2}\left(\delta-C_{0}\right)^{2}+C_{T H S} T H S+C_{\alpha} \alpha$

$C_{\delta}=C_{1} P_{d}, \quad C_{\delta 2}=C_{2} P_{d}, \quad C_{T H S}=C_{3} P_{d}, \quad C_{\alpha}=C_{4} P_{d}$

$C_{1}=C_{11}(M)+C_{12}(M) M, \quad C_{2}=C_{21}(M)+C_{22}(M) M$,

$C_{3}=C_{31}(M)+C_{32}(M) M, \quad C_{4}=C_{41}(M)+C_{42}(M) M$

where the $C_{\star \star}$ coefficients are assumed, for simplicity, piecewise constant between Mach numbers of 10 different values, spanning the flight domain. The coefficients are obtained using polynomial regression, with least-squares fit on a grid of parameter points.

The dynamic part of the elevator model is a function of the elevator actual deflection $\left(\delta_{e}\right)$, aerodynamic force $\left(F_{\text {aero }}\right)$, and sign of elevator speed $\left(\operatorname{sign}\left(\dot{\delta}_{e}\right)\right)$, which makes the plant a parameter dependent switched LPV system with three scheduling variables. Different linearization techniques were applied to the nonlinear actuator model, but standard techniques were not able to capture the plant response properly. Responses of the models obtained with analytical linearization (blue), and small perturbation method (red dashed) are shown in Fig. 5. The small perturbation method,

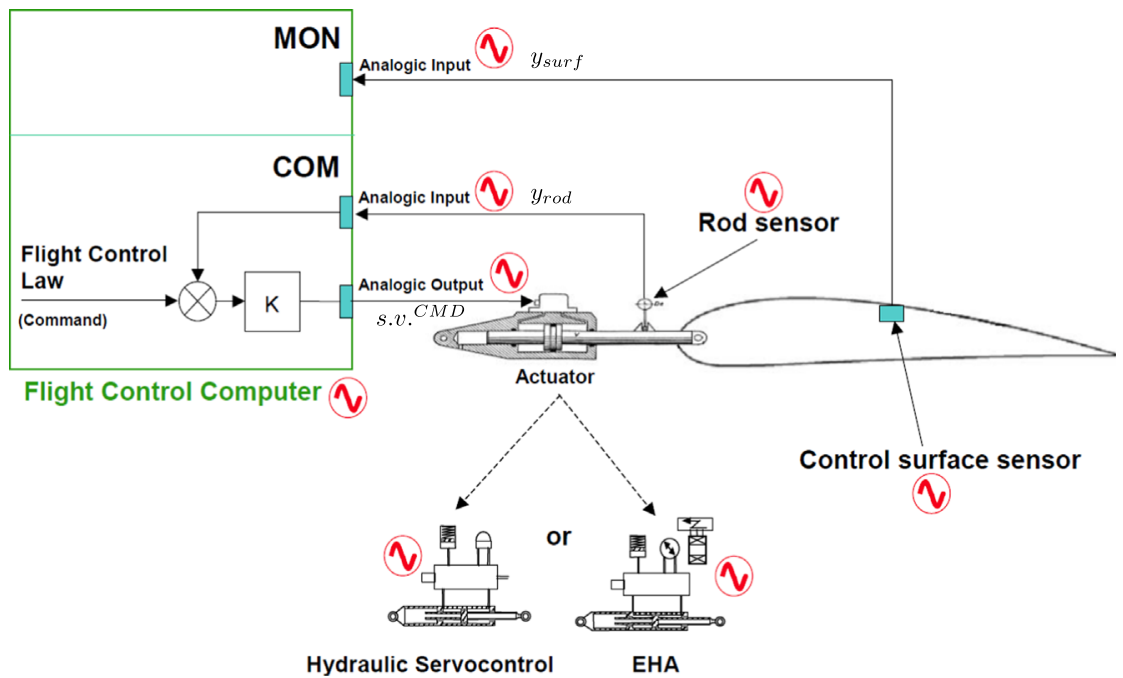

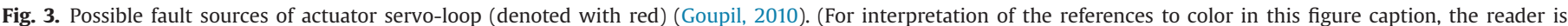
referred to the web version of this paper.) 


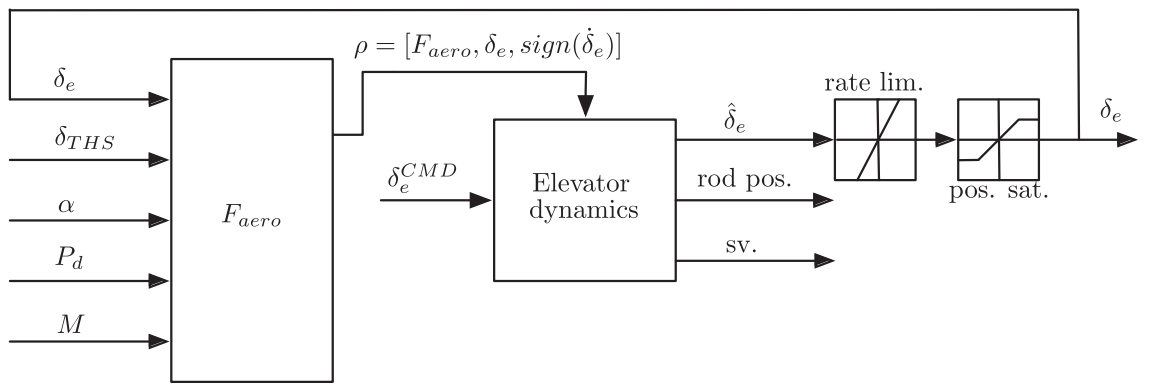

Fig. 4. Model structure of the elevator actuator model, static nonlinearity of $F_{\text {aero }}$ on the left, actuator dynamics on the right.

with a given initial deflection and constant aerodynamic force, is not able to reproduce either the positive or the negative variance from the trim point, while the analytical linearization fails only in the positive deflection case. Despite the unsuccessful efforts it is possible to obtain the structure of the underlying discrete time dynamical system:

$x_{k+1}=\left[\begin{array}{cc}1 & p_{1}(\rho) \\ 1 & 0\end{array}\right] x_{k}+\left[\begin{array}{c}p_{2}(\rho) \\ 0\end{array}\right] u_{k}$

$y_{k}=\left[\begin{array}{cc}p_{3}(\rho) & 0 \\ 1 & 0 \\ 0 & 2\end{array}\right] x_{k}+\left[\begin{array}{c}0 \\ 0 \\ p_{4}(\rho)\end{array}\right] u_{k}+\left[\begin{array}{l}1 \\ 0 \\ 0\end{array}\right] f_{k}$

where the structure of the model is unchanged in every parameter point, only the parameter values of $p_{1}, p_{2}, p_{3}, p_{4}$ are scheduled with $\rho=\left[F_{\text {aero }}, \delta_{e}, \operatorname{sign}\left(\dot{\delta}_{e}\right)\right]$, leading to a discrete time LPV system (sampling time is set to $0.01 \mathrm{~s}$ ). Grey-box identification (Ljung, 1999) based on the parameter estimation method (PEM) is used to obtain the values of $p_{1}, p_{2}, p_{3}, p_{4}$ parameters at different scheduling variable values of $F_{\text {aero }}=[-10000: 2000: 10000] N, \quad \delta_{e}=$ $\left[0.9 \delta_{e, \min }: 0.1\left(\delta_{e, \min }+\delta_{e, \max }\right): 0.9 \delta_{e, \max }\right], \quad \operatorname{sign}\left(\dot{\delta}_{e}\right)=[-1 ; 1]$, representing a large part of the actuator operating domain. Using the LTI models obtained with PEM (green dotted line in Fig. 5) almost perfect match can be observed with the nonlinear response (denoted with black). Using the LTI models at frozen parameter values, the system can be casted into a polytopic LPV actuator model. The model augmented with position and rate saturation, coupled with the $F_{\text {aero }}$ scheduling parameter model, represents the nonlinear model very accurately in a large part of the operating envelope.

\section{LPV FDI filter design for the aircraft}

To compare the local and global model based approaches, the two FDI filters are applied to the same aircraft model and cross compared with each fault type to assess their detection performance, including true detection, false alarm and missed detection rates. The false alarm statistics is further challenged with offnominal flight scenarios, when elevator faults are occurring during the assessment of aileron FDI, and aileron faults occurring during elevator assessment.

\subsection{LPV FDI for aileron}

A geometric LPV FDI filter (Szaszi et al., 2005) is designed for the left inner aileron fault detection problem of the aircraft, which is augmented with an $\mathcal{H}_{\infty}$ post filter to shape the residual. As described above, load factor measurements are omitted from the model, since the $D$ matrix associated with these outputs is nonzero, which makes the geometric FDI synthesis more complicated. The resulting design model has 9 outputs, 15 inputs (including the three fault directions and three wind gust disturbance directions) and 27
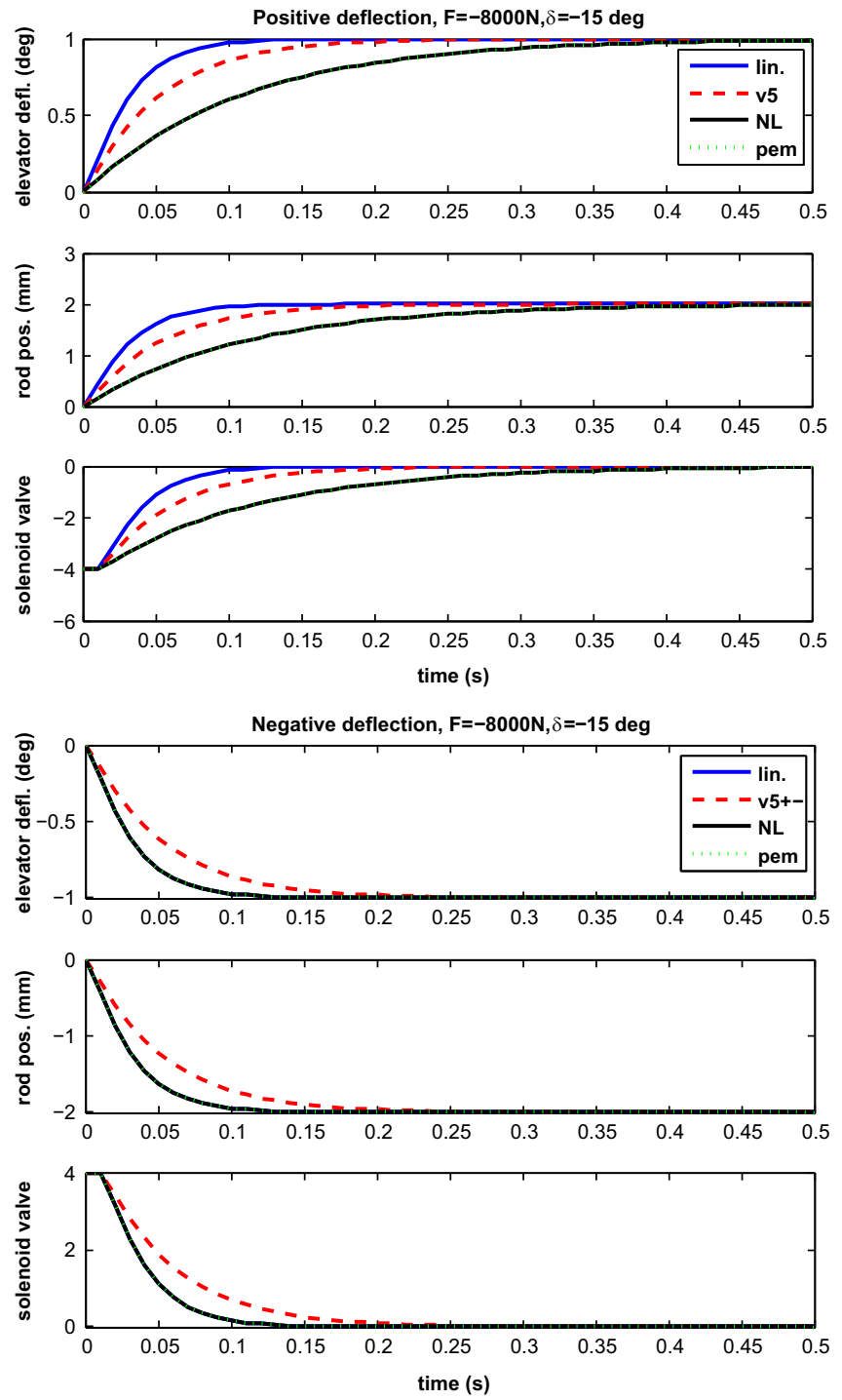

Fig. 5. Step response of elevator models obtained with different linearization techniques and the nonlinear behavior, positive deflection (upper three), and negative (lower three). (For interpretation of the references to color in this figure caption, the reader is referred to the web version of this paper.)

states, a relatively high state order includes actuator and sensor dynamics. The LPV design model is scheduled with calibrated airspeed and altitude, since after careful evaluation of the system dynamics, mass and c.g. position are omitted from the scheduling variables, due to their less significant effect on the dynamics related to aileron FDI.

The baseline geometric (FPRG) FDI filter obtained using the methods developed in Balas et al. (2003) has 7 residual outputs, 
18 inputs, and 18 states. Since perfect decoupling is possible, the parameter dependent transfer functions between elevator fault to residuals and rudder fault to residuals are zero, while the residuals all have nonzero response for aileron faults. To be able to augment the FPRG filter with an $\mathcal{H}_{\infty}$ post filter using an output injection, as shown in Fig. 1, the original 18 inputs of the system are augmented by 18 additional inputs, each of them directly acting on one of the 18 states of the original FPRG filter. To pose an $\mathcal{H}_{\infty}$ optimization problem for the post filter, a frozen point LTI plant from the LPV aircraft model with a frozen point FPRG filter is augmented with the design weights, as shown in Fig. 6. The list of design weights are the following: $W_{u}$ represents the uncertainty weights, $W_{d r}$ is responsible for the Dryden wind gust disturbance, sensor noise is filtered through $W_{d}$, while fault tracking error is penalized by $W_{e}$ and for well-posedness the pseudo-control signals of the $\mathcal{H}_{\infty}$ filter are penalized by $W_{p}$. Fault tracking is achieved only in a limited frequency range described by $T_{i d}$.

The weights are chosen according to the following logic:

- $W_{u}=\operatorname{diag}\left(0, w_{a}, w_{a}, w_{a}, w_{a}, w_{s p}, w_{e}, w_{r}, w_{e}\right)$ corresponds to the input uncertainty weights on the 9 input channels, where $w_{a}=$ $1.5(s+4) /(s+160)$ is the Aileron, $w_{e}=0.75(s+20) /(s+200)$ is the elevator and THS, $w_{r}=0.75(s+20) /(s+200)$ is the rudder, $w_{s p}=2.25(s+4) /(s+160)$ is the spoiler uncertainty respectively, where the elevator and the rudder have the lowest amount of uncertainty, followed by ailerons and the highest value is associated with spoilers, while engine inputs are considered perfect in the present investigation. Higher amount of uncertainty is assumed at higher frequencies corresponding to less knowledge of the system dynamics in that frequency range.

- $\left\|\Delta_{a}\right\|_{\infty} \leq 1$ is a norm bounded structured uncertainty block on the input channels, with the structure of $\operatorname{diag}\left(0, \Delta_{\text {ail }}^{4 \times 4}, \Delta_{s p}^{1 \times 1}\right.$, $\left.\Delta_{\text {tail }}^{3 \times 3}\right)$, representing uncertainty on ailerons, spoilers, and tail surfaces respectively.

- $W_{d r}$ is the moderate Dryden wind gust filter (probability of exceedance is $10^{-3}$ ), according to MIL-HDBK-1797, with 240 kts velocity and 533.4 feet wingspan.

- $W_{d}=0.05 I_{9}$ is a noise weighting filter on all 9 measurement channels, corresponding to $0.05 \mathrm{rad}$ and $0.05 \mathrm{rad} / \mathrm{s}$ noise for magnitude and rate sensors respectively.

- $T_{i d}=0.25 /\left(s^{2}+s+0.25\right)$ is the ideal fault tracking behavior, requiring a second-order $0.5 \mathrm{rad} / \mathrm{s}$ response which is tuned to achieve a tradeoff between detection speed and disturbance attenuation.

- $W_{e}=1 /(s+1)$ is a low pass filter which ensures fault response tracking up to $1 \mathrm{rad} / \mathrm{s}$, penalizing the difference between the ideal fault reconstruction and the residual response.

- $W_{p}=0.1 I_{18}$ is a performance weighting function penalizing the outputs of the $\mathcal{H}_{\infty}$ filter with weights on all pseudo-control channels, required for well-posedness of the optimization.

The weighted interconnection with the FPRG filter included has 29 states, 26 outputs and 43 inputs, and the resulting $\mathcal{H}_{\infty}$ FDI filter,

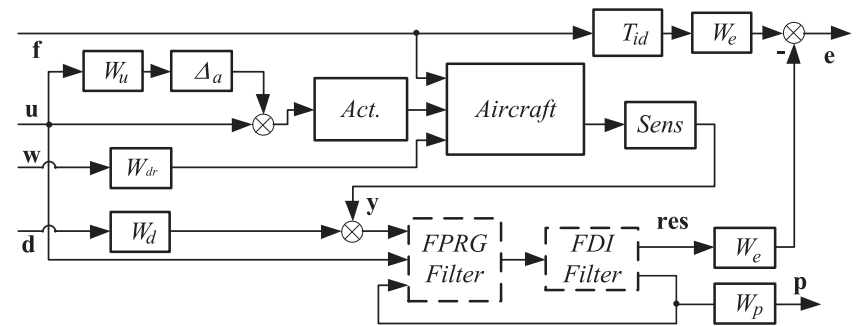

Fig. 6. Input multiplicative uncertainty case, weighted interconnection for $\mathcal{H}_{\infty}$ filter synthesis. not considering the effects of uncertainty $\Delta_{a}$, has 7 measurements and 19 control outputs. Combining the nominal LTI FPRG filter and the LTI post filter results in a 30 states, 18 input and 1 output filter, after computing minimal realization. In the benchmark simulation the 18 states of the LPV FPRG filter are augmented with the 29 states of the $\mathcal{H}_{\infty}$ FDI post filter. It is important to note that a LPV controller synthesis for the post filter would be computationally too expensive. A good engineering compromise is made by combining the algebraic solution for the LPV FPRG filter with an LTI $\mathcal{H}_{\infty}$ filter to provide an LPV residual generator with reduced computational complexity.

To analyze the performance of the aileron LPV FDI filter, it is applied to the nonlinear aircraft model after taking the trim values into consideration, on both control input and sensor output signals. Since the simulation is implemented under Simulink with a $0.01 \mathrm{~s}$ fixed step size, the corresponding filters are also discretized with the same sampling time using bilinear transformation. It is also worth mentioning that the simulation is in closed-loop with the flight control system set to altitude and heading hold mode and moderate atmospheric wind gust disturbances are perturbing the aircraft flight. For threshold selection purposes the filter is applied to the nonlinear aircraft model at various cruise conditions with appropriate trim scheduling with elevator faults, to test the fault isolation properties. The left elevator drifts from the commanded position with $5 \% \mathrm{~s}$ rate starting at $20 \mathrm{~s}$. The simulation starts from a typical flight condition of $240 \mathrm{kts}$ and $26000 \mathrm{ft}$, the c.g. position is $x_{c g}=0.3$ and the weight is $200000 \mathrm{~kg}$, the data is normalised with physical deflection limits on control surfaces and with maximum achieved aerodynamic angles and angular rates in measurements, due to Airbus confidentiality reasons. The change in aircraft behavior is clearly noticeable, a large pitch excursion can be seen in Fig. 7, while the flight control system counteracts with the adjacent elevator.

The LPV FDI residual reaches a value of 0.105 during the manoeuvre, as seen in Fig. 8, indicating good fault decoupling properties, hence the detection threshold is set with a safety factor of $1.5-0.105 \times 1.5$. The detection performance is analyzed on a left inboard aileron jamming scenario, resulting in a bias on the rod sensor at $20 \mathrm{~s}$ shifting the surface off from the commanded position with a fixed constant value. The flight is at a representative cruise condition, with the moderate Dryden wind gust, and off from the nominal FDI design condition: $V_{C A S}=200 \mathrm{kts}$ and $h=23000 \mathrm{ft}, x_{c g}=0.3$, and $m=200000 \mathrm{~kg}$. The detection time performance (relative to the performance specification of Airbus) of 0.00508 is achieved with the LPV method and the fault reconstruction performance is excellent, as shown in Fig. 9. Frozen point LTI cases show a similar performance at the nominal design point but their performance quickly degrades as flight parameters are departing from the design point, clearly indicating the advantage of the LPV method.

The results are also validated in an industrially relevant functional engineering simulator (FES) by Deimos Space S.L.U., where the filter performance is assessed in a rigorous simulation campaign, on a grid of flight envelope with $\pm 10 \%$ uncertainty in scheduling parameters of velocity, mass, altitude and c.g. position, and in addition $\pm 5 \%$ uncertainty in aerodynamic coefficients. 324 simulations are analyzed in total, where 158 cases do not lead the aircraft out of the valid flight envelope, and the filter achieved 0.0074 mean and 0.0314 maximum detection time performance, while the true detection is $100 \%$, with no false alarms and missed detections. Fig. 10 shows the spread of filter residual outputs (all of them normalised). Notice that the constant detection threshold derived above has to be changed to an adaptive threshold scheduled with a roll rate $(p)$, which uses a simple lookup table as shown in Fig. 15, with low values at a low roll rate and higher threshold at higher absolute values of the roll rate. 

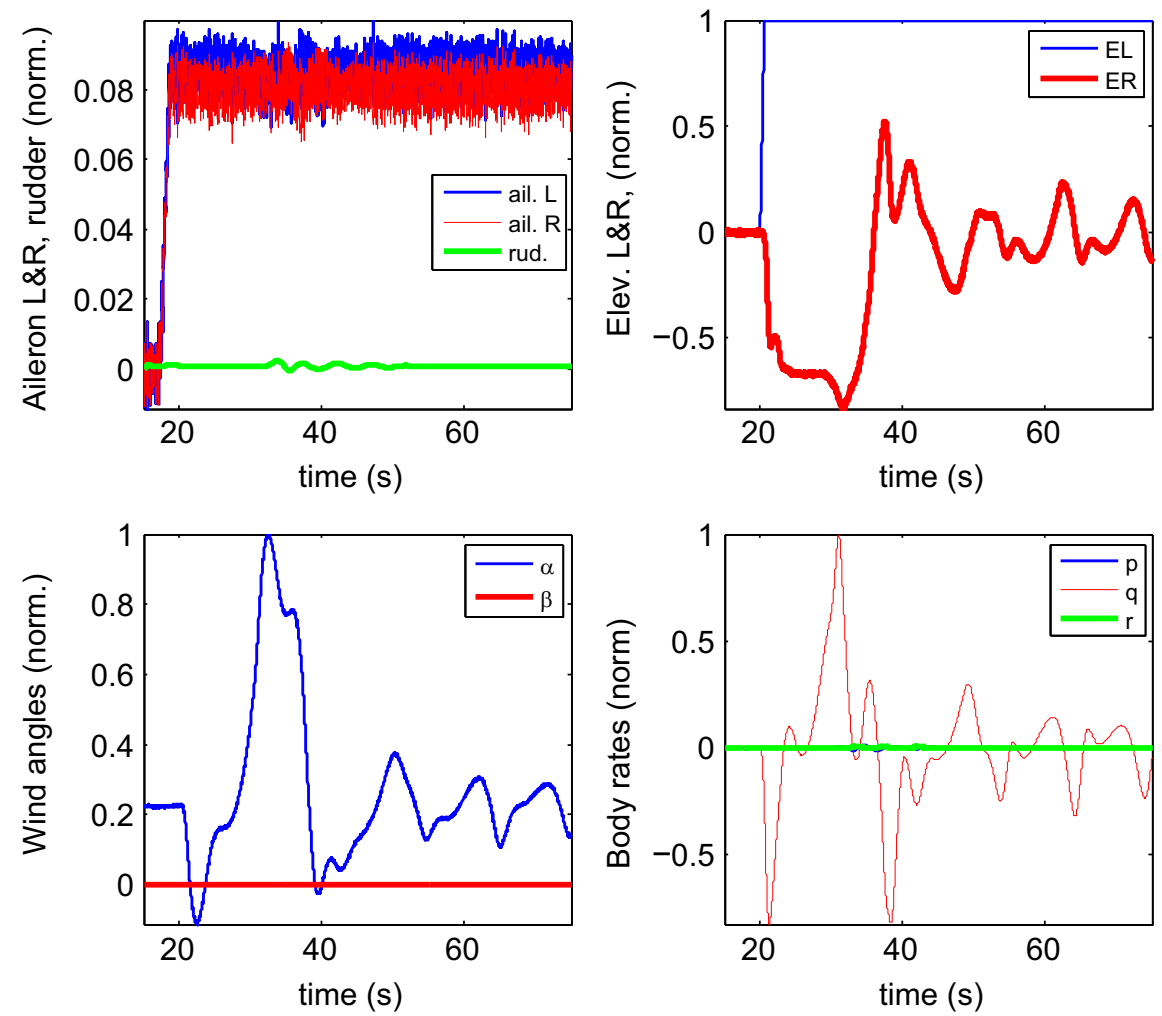

Fig. 7. Left elevator runaway scenario ( $V_{C A S}=240 \mathrm{kts}, h=26000 \mathrm{ft}, x_{c g}=0.3$ and $m=200000 \mathrm{~kg}$ ), aircraft response, fault occurs at $20 \mathrm{~s}$.
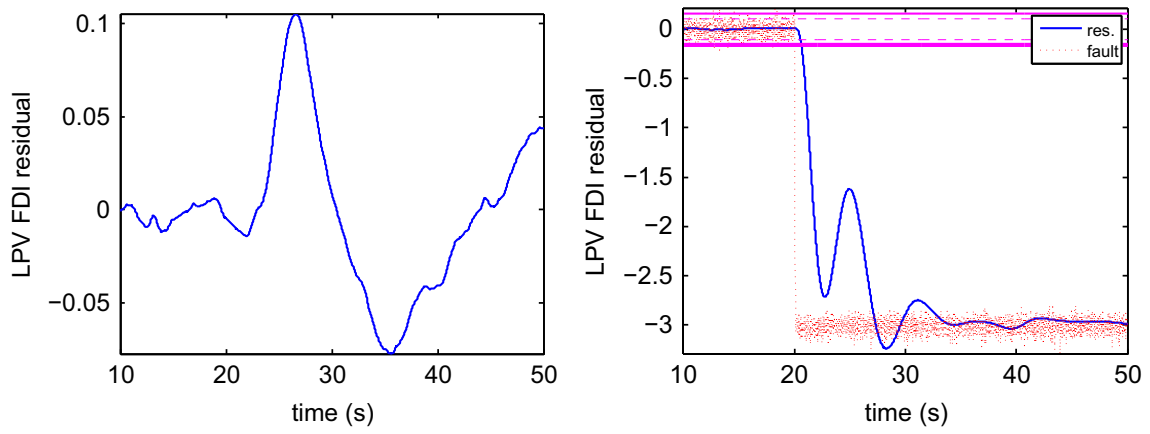

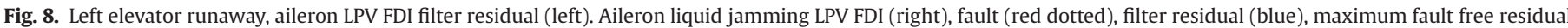

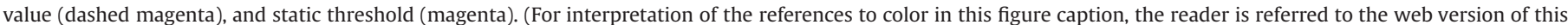
paper.)

The raw adaptive threshold is passed through a low pass filter of $F_{\text {thrs,ail }}=1 /\left(s^{2}+2 s+1\right)$ to avoid rapid changes, before compared with the residual to form the fault diagnostic boolean value.

The ultimate test of the design is performed by Deimos Space (project coordinator and industrial partner) using a more sophisticated FES capable of benchmarking all the consortium designs in equivalent conditions. A true Monte-Carlo campaign evaluated the shortcomings of each design where 2200 valid runs are decomposed into two main cases: 1200 fault-free runs distributed evenly among six benchmark-defined flight manoeuvres and 1000 runs with faults at the flight manoeuvre for the selected fault scenario including solid-, liquid-jamming and disconnection (Goupil \& Marcos, 2012). The FPRG LPV design for the aileron scores a detection performance maximum of 0.139 , well below the specification of 1 , with no false alarms and missed detections. These results are obtained after one iteration of the implementation, since the first delivery of the code to Deimos Space contained a software bug.

\subsection{FDI filter design for the elevator}

Simple parity relations can be formed using the elevator actuator dynamics as described in Eq. (21). The main assumption about the elevator runaway (or jamming) fault is that it acts on the first measured output only, which using Eq. (21) leads to

$y_{1}=p_{3}(\rho) x_{1}+f_{1}$

while $y_{2}, y_{3}$ are unchanged and independent of $f_{1}$, hence can be used as parity equations. The fault acting on the plant can be expressed as

$x_{2}=0.5\left(y_{3}-p_{4}(\rho) u\right)$

$f_{1}=y_{1}-p_{3}(\rho) x_{1}$

Introducing the estimate of $\hat{x}_{1}=\xi_{1}$, still independent of $f_{1}$, leads to the relation of $\hat{y}_{1}$, from which a residual $\left(r_{e}\right)$ can be formed:

$\hat{y}_{1}=p_{3}(\rho) \xi_{1}$ 

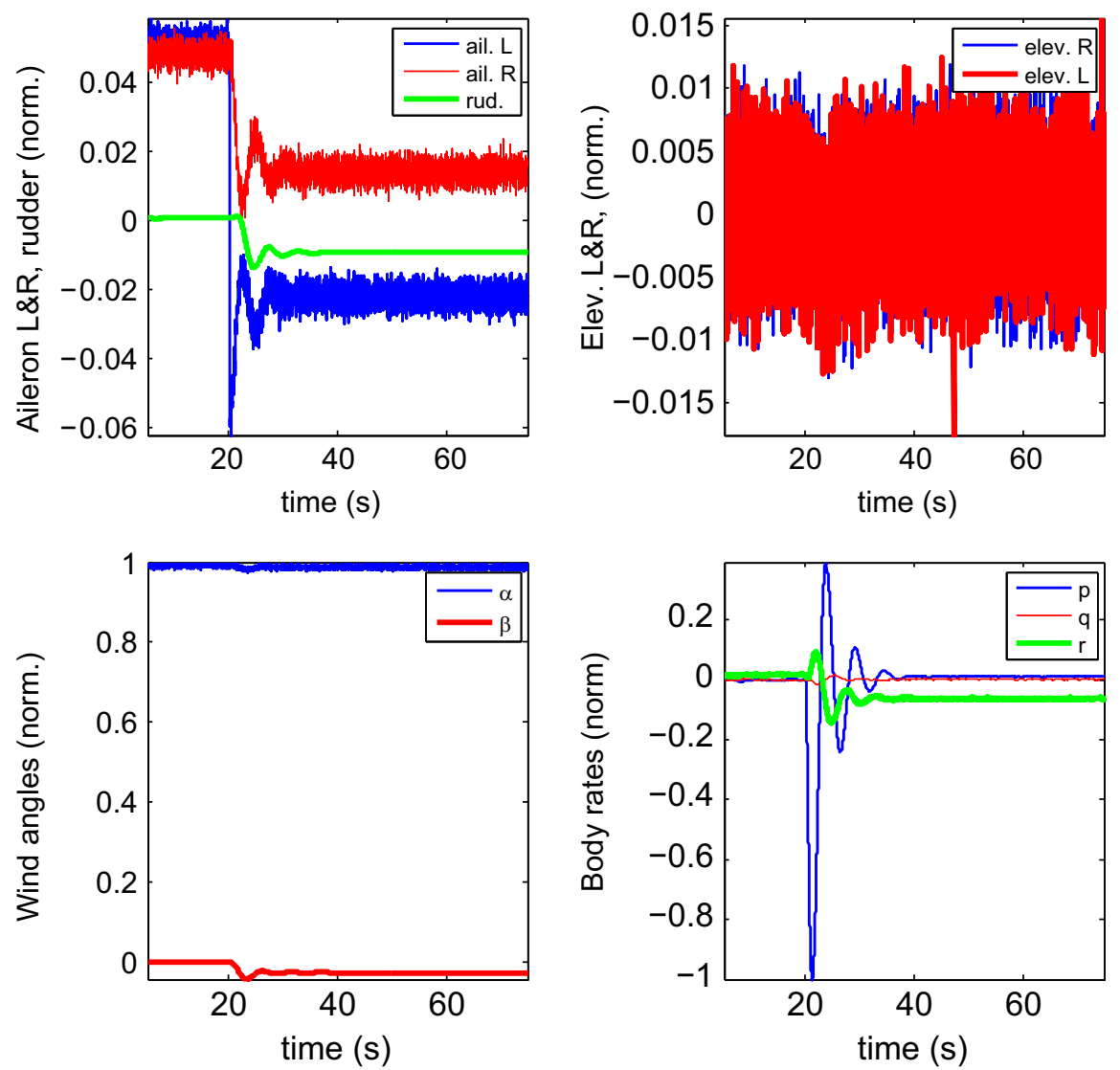

Fig. 9. Left aileron liquid jamming scenario ( $V_{C A S}=200 \mathrm{kts}, h=23000 \mathrm{ft}, x_{c g}=0.3$ and $m=200000 \mathrm{~kg}$ ), aircraft response, fault occurs at $20 \mathrm{~s}$.

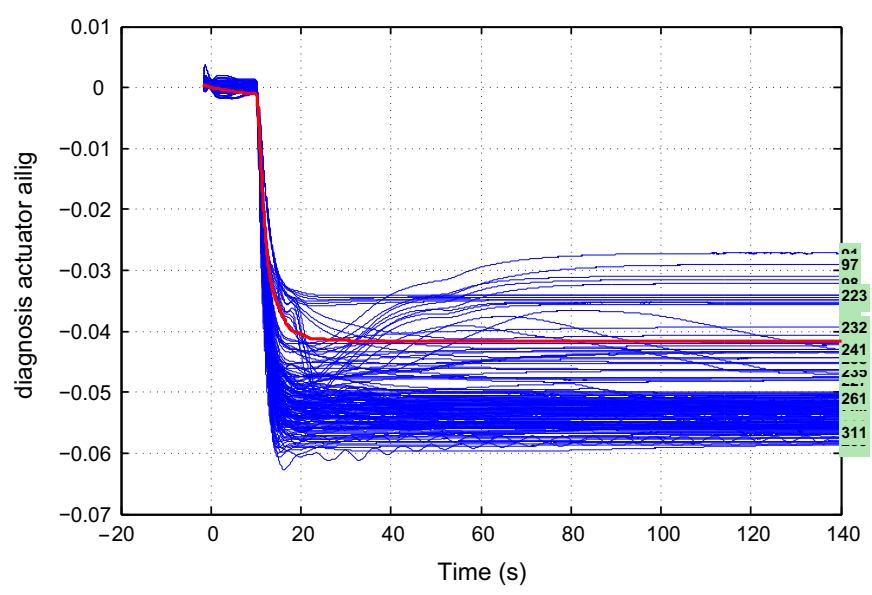

Fig. 10. Filtered residual: aileron liquid jamming scenario, nominal behavior with red and parametric run of 324 cases with blue (case number on the right), fault occurs at $10 \mathrm{~s}$. (For interpretation of the references to color in this figure caption, the reader is referred to the web version of this paper.)

$\dot{\xi}_{1}=\xi_{1}+p_{1}(\rho)\left(0.5 y_{3}+0.5 p_{4}(\rho) u\right)+p_{2}(\rho) u-\lambda\left(\xi_{1}-y_{2}\right)$

$r_{e}=y_{1}-p_{3}(\rho) \xi_{1}$

where all three independent measurements are used to form the residual, by introducing a reduced order state estimator for the system. Since the system equations are used directly, the $p_{\star}(\rho)$ parameters depending on $F_{\text {aero }}, \delta_{e}, \operatorname{sign}\left(\dot{\delta}_{e}\right)$ determine the filter equations, forming an LPV observer. Only one design parameter $(\lambda)$ has to be chosen to achieve the desired detection time, which complies with industrial practice to use tuning knobs which have physical meaning and to have the fewest set of design tuning parameters. Since the proposed model does not include measurement noise, but it is present in the real world system, it is necessary to filter the residual $\left(r_{e}\right)$, with an appropriately chosen low pass filter, which is selected conservatively to $F_{\text {res,elev }}(S)=$ $(0.1 /(s+0.1))^{2}$. The last step in the detection framework is to set the threshold on the residual magnitude for fault declaration, which is selected based on a fault isolation problem, where the aileron is faulty and the elevator FDI should not declare a false alarm (assuming a safety factor of $50 \%$ in the elevator FDI case also). The proposed design has only 3 tuneable design parameters which is very favorable from industrial point of view.

The scenario under investigation is elevator jamming at $0^{\circ}$ position in a turn coordination manoeuvre. The elevator commands at the same nominal design point $\left(V_{C A S}=240 \mathrm{kts}\right.$, $h=26000 \mathrm{ft}, x_{c g}=0.3$ and $m=200000 \mathrm{~kg}$ ) are shown in Fig. 11, which indicates a gentle reaction from the flight control system, since unlike in a runaway case the consequences of jamming are not recognized immediately. The opposite side counteracts the effect of the fault, but if the initial deflection of the elevator is also around $0^{\circ}$, the fault remains unobservable, until a longitudinal maneuver starts. The performance of the filter is assessed in a similar simulation campaign described above, at different flight envelope points, with $10 \%$ uncertainty on scheduling parameters and $5 \%$ uncertainty on aerodynamic coefficients. The simulation consists of 158 valid runs, where the flight control commands spread a wide range, posing a difficult task to simple filtering techniques with various initial conditions and transient behaviors. In certain situations the elevator actuator is reaching its physical deflection limit when a demanding pitching maneuver is commanded, hence it is important to augment the LPV model with the saturation effects. Note that all the figures are normalized due to Airbus confidentiality reasons. 

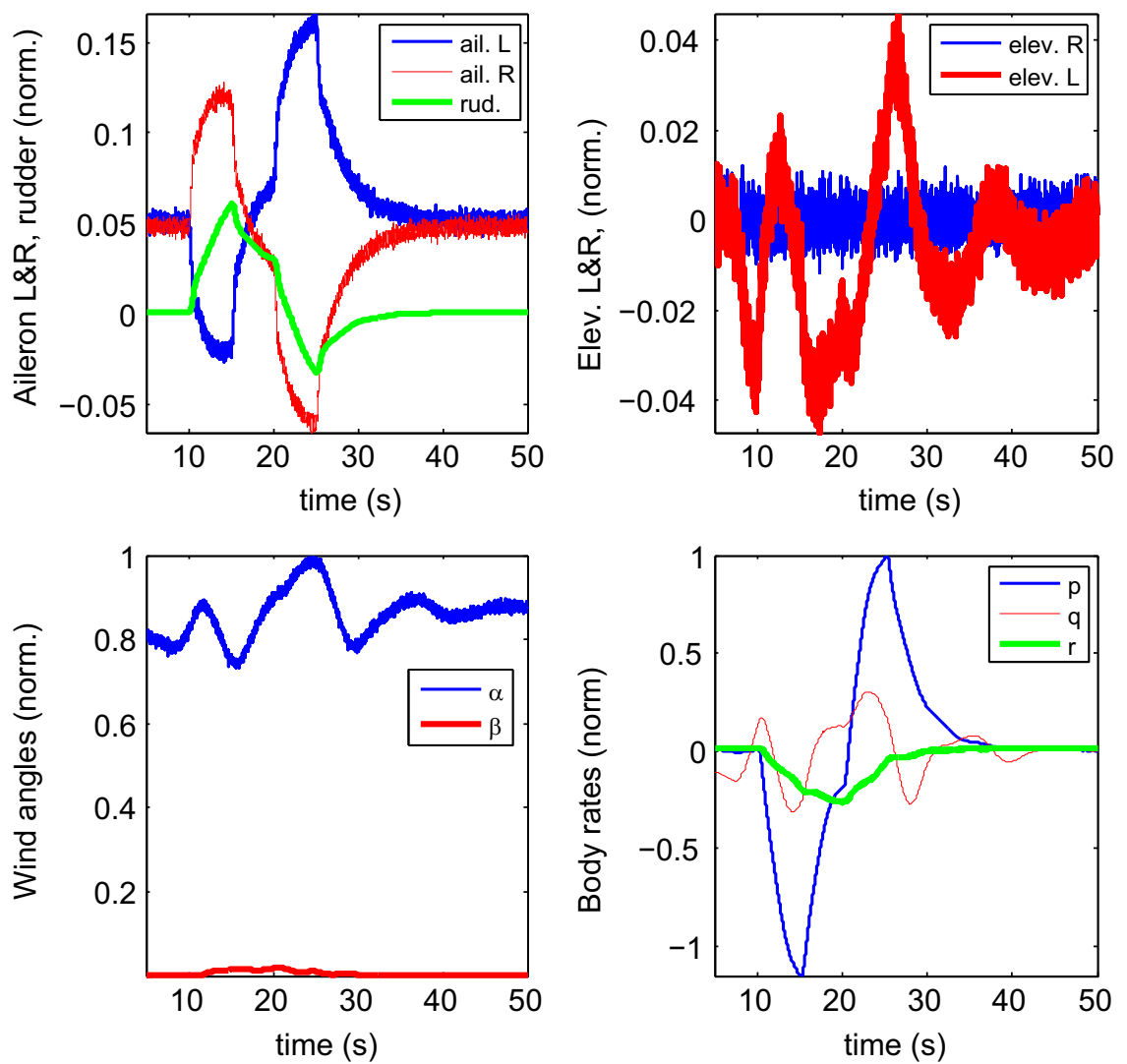

Fig. 11. Right elevator jamming scenario ( $V_{C A S}=240 \mathrm{kts}, h=26000 \mathrm{ft}, x_{c g}=0.3$ and $m=200000 \mathrm{~kg}$ ), aircraft response at a single flight point, fault occurs at $8 \mathrm{~s}$.

The measurements of elevator deflection in the jamming scenario are shown in Fig. 12. The nominal flight envelope trim point is plotted with red, while the parametric simulation runs are plotted with blue, where a wide spectrum of initial actuator deflections can be seen up to $8 \mathrm{~s}$, when suddenly the elevator shifts to $0^{\circ}$ deflection, suffering from a jamming fault. It is intuitive to see the occurrence of the fault given the large number of simulation runs, however in the less extreme cases the initial and faulty deflections are within the bounds of the measurement noise. Elevator rod position measurements are shown in Fig. 13. Signals in the jamming case are closer to neutral position than the signals if a runaway fault would occur, making jamming far more challenging to detect. Although in the nominal design case a static threshold is selected, in the more rigorous systematic simulation campaign the threshold is changed to an adaptive one, which is deflection command ( $\delta_{e}^{C M D}$ ) dependent (see Fig. 15). This is necessary, since at larger deflections the model fidelity is lower and saturation effects might influence the precision also. To address the problem, the threshold is held constant around small elevator commands and then ramps up to higher values as commands are reaching the physical limits of the actuator. In addition, to avoid rapid changes, the output of the threshold function is filtered with the a low pass filter of $F_{\text {thrs,elev }}=0.2 /(s+0.2)$.

The fault detection time in the elevator jamming case is longer than in the aileron liquid jamming or runaway cases, since the elevator is not deflected from the trim position until a manoeuvre starts, which hides the effect of the fault. The residual response, shown in Fig. 14 is more spread out and fault detection only occurs when the aircraft is subjected to a manoeuvre requiring elevator movement. Faults are detected in all 158 valid cases, with zero false detection and missed detection rate is also zero, achieving the required $100 \%$ true detection rate. The minimum detection time performance is 0.0124 , while the maximum is 0.049 . The large variation is due to the fact that the elevator is barely used from $8 \mathrm{~s}$ to $10 \mathrm{~s}$ in most of the cases, until the maneuver starts, and

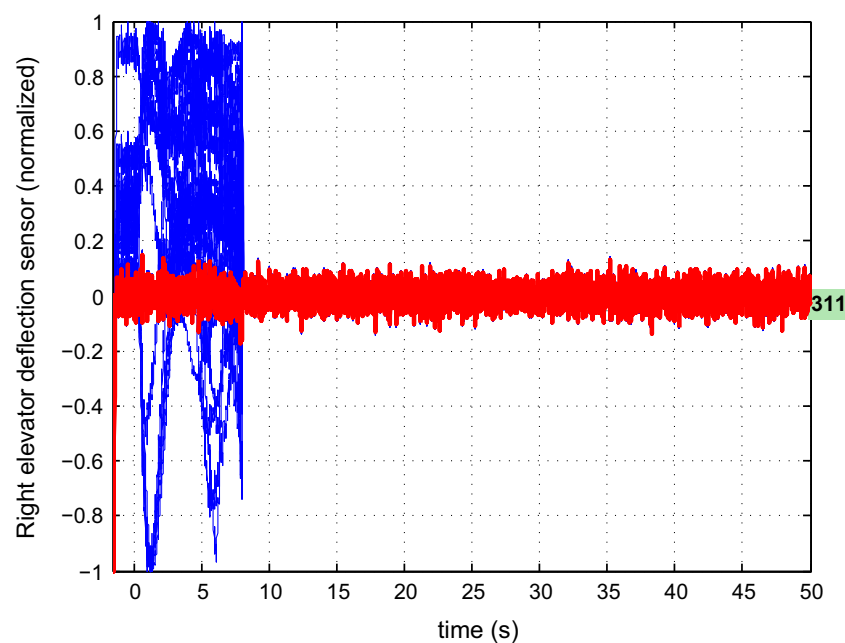

Fig. 12. Elevator deflection sensor: right elevator jamming scenario, nominal behavior with red and parametric run of 324 cases with blue (case number on the right), fault occurs at $8 \mathrm{~s}$. (For interpretation of the references to color in this figure caption, the reader is referred to the web version of this paper.)

the difference between the original deflection and the faulty deflection with jamming is minimal. However, since the jamming scenario is less critical than runaway, longer detection time is allowed according to the specifications, which is the reason of excellent detection time performance.

The ultimate test of the design for the elevator FDI is performed similarly to the aileron FDI assessment, by Deimos Space using a benchmarking FES on a set of 2200 valid runs decomposed into two main cases: 1200 fault-free runs distributed evenly among six benchmark-defined flight maneuvers and 1000 runs with faults at the default flight maneuver for the selected elevator jamming fault 


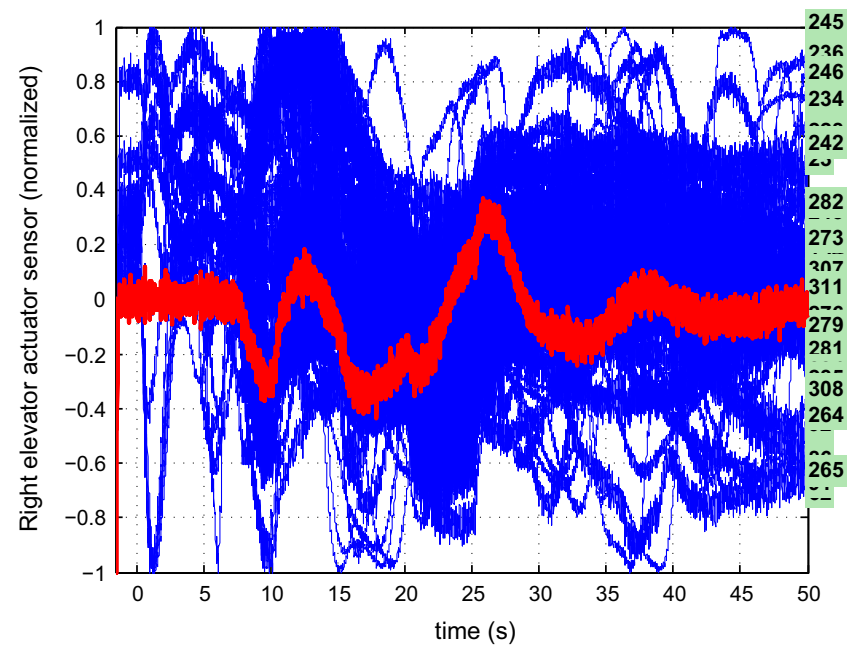

Fig. 13. Elevator rod position: right elevator jamming scenario, nominal behavior with red and parametric run of 324 cases with blue (case number on the right), fault occurs at $8 \mathrm{~s}$. (For interpretation of the references to color in this figure caption, the reader is referred to the web version of this paper.)

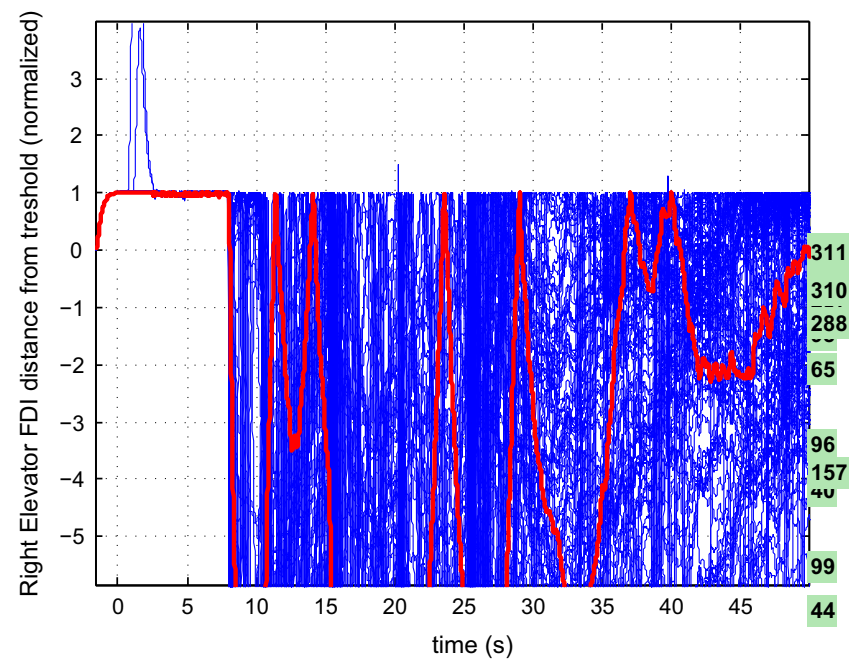

Fig. 14. Residual-threshold difference: right elevator jamming scenario, nominal behavior with red and parametric run of 324 cases with blue (case number on the right), fault occurs at $8 \mathrm{~s}$. (For interpretation of the references to color in this figure caption, the reader is referred to the web version of this paper.)

at $0^{\circ}$. The LPV detection filter based design for the elevator scored a detection performance maximum of 0.046 , well below the specification of 1 , with no false alarms and missed detections. These results are obtained after no iteration of the implementation showing the benefit of advanced model-based design.

\section{Implementation aspects of LPV FDI filters}

The main difficulty in designing highly complex algorithms for fault detection lies in the implementation, since all software implemented on the flight control computers (FCC) has to be certified. Most often the FDI filters are designed in continuous time, with limited attention to real-time aspects and relying on strict modeling assumptions such as perfect knowledge of trim values, no quantization and delay on signals. On the other hand the FCCs have limited computational capability and the filters might need some tuning on-site by an engineering expert, especially with respect to their threshold functions. For this reason, it is advantageous if a design method have tuning knobs with which the performance can be tuned according to the specifications in a transparent manner, without full knowledge of the underlying theory.

In the final implementation, when the theory is applied to a real world, highly complex, fully nonlinear system the original design assumptions often fail and more conservative approaches or additional tuning parameters have to be introduced. The thresholding functions in both local and global approaches have to be scheduled, as described above, and increased leading to more conservative results, to accommodate for the differences in model fidelity around different points of the operating envelope. The choice of adaptive threshold with the appropriate scheduling variable and magnitude of it across the scheduling range requires engineering insight and trial and error type tuning. Hence the number of tuning parameters in the two cases are difficult to estimate, since important design choices have to be made regarding the model generation and sometimes, in the weighting functions even the number of parameters are a part of the design decisions. But it is safe to say that the global model based method requires significantly more engineering insight and roughly 20 tuning parameters, while the detection filter based local method requires only tuning of 10 design variables.

It is also important to note that LTI and LPV models require careful trim routines and in the final implementation not only the detection filter matrices have to be scheduled with the flight parameters, but also the measurements and commands have to be compensated with their trim values, which are available from the flight control system. Further difficulty is to be compliant with the Aerospatiale graphic language SAO (Specification Assistee par Ordinateur) (Briere \& Traverse, 1993; Spitzer, 2001). Software algorithms have to be coded with a limited set of elementary blocks. This reduces the precision of the computations, for example in the elevator FDI case, the parameter dependent matrices have to be interpolated with a $6 \times 6$ lookup-table. Implementation aspects have to take into account discrete switching behavior, often encountered in highly complex architectures such as the fly-by-wire system, related to managing flight control system resources. In initial cold start of the FCCs the filter residuals require at least 2-3 s to settle down, while the fault detection residual must be set to zero artificially. A similar transient behavior has to be handled in cases when one FCC becomes active from standby mode, or when the flight control law switches from normal to alternate law.

As stated above both global and local detection methods achieved full detection performance, with no false alarms and no missed detections. Their detection time performance characteristics differ only due to the different specifications on the aileron and elevator scenarios. Their fundamental difference lies in the implementation aspects, since both filters have to be coded with SAO sheets and the engineers implementing them on flight certified hardware would like to be aware of the number of tuning parameters and their effect on the performance. The implementation of the local FDI algorithm with SAO blocks uses 75 elementary blocks, while the execution time is $21 \%$ of the allocated maximum. On the other hand the complex global model based FDI filter uses 2159 elementary blocks and the execution time is $459 \%$ of the maximum allowed, which seems high, but there is a clear need for global model based methods, since certain FDI problems cannot be solved with local methods due to the lack of analytical redundancy. Hence, within the next generation of FCCs the available computational power have to increase to take into account the computational needs of FDI methods.

A similar design decision is the sampling time of the implementations. Using a $50 \mathrm{~Hz}$ version of the elevator FDI filter showed 0.0496 maximum detection time performance (vs. 0.049 of the original $100 \mathrm{~Hz}$ one), but the $25 \mathrm{~Hz}$ version has unacceptable performance with higher than 1 maximum detection time performance. 

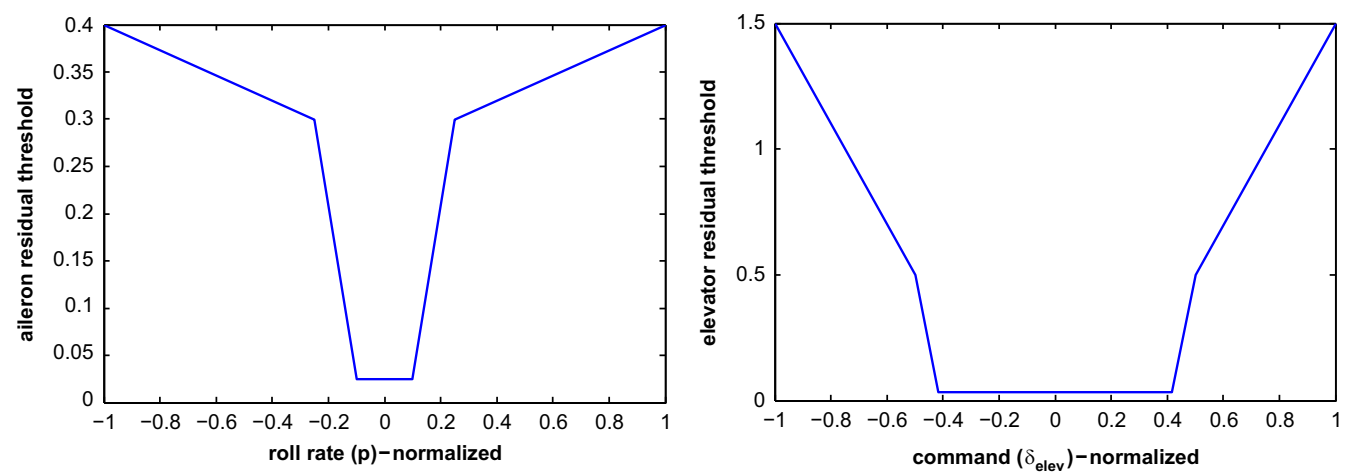

Fig. 15. Aileron residual lookup table as a function of roll rate (left). Elevator residual lookup table and function of elevator command (right).

\section{Conclusion}

The present paper detailed the development steps of two inherently different design methods to detect aircraft actuator faults. Although the methods are different, their final industrial evaluation resulted in a similar performance. The main difference between them is the computational load, which clearly favors the local model based methods. However in many situations when a limited set of measurements are available, the local methods may be infeasible and the present paper shows that even a method based on a 6-DOF aircraft model with a high number of states can be implemented with a reasonable amount of software code, and despite its complexity can perform flawlessly in the industrial evaluation, while the computational capabilities of the next generation of flight control computers will enable the use of such methods.

\section{References}

Alwi, H., Edwards, C. \& Marcos, A. (2010). Actuator and sensor fault reconstruction using an LPV sliding mode observer. In AIAA guidance, navigation, and control conference. Toronto, Canada.

Andrieu, L. (1999). A340-600: Equations caracteristiques et donnees numeriques des servocommandes. Technical report, Airbus.

Balas, G., Bokor, J., \& Szabo, Z. (2003). Invariant subspaces for LPV systems and their applications. IEEE Transactions on Automatic Control, 48(11), 2065-2069.

Bokor, J., \& Balas, G. (2004). Detection filter design for LPV systems-A geometric approach. Automatica, 40, 511-518.

Briere, D., \& Traverse, P. (1993). Airbus A320/A330/A340 electrical flight controls-A family of fault-tolerant systems. In 23rd international symposium on faulttolerant computing, FTCS-23 (pp. 616-623). Toulouse.

Chang, C. B., \& Athans, M. (1978). State estimation for discrete systems with switching parameters. IEEE Transactions on Aerospace and Electronic Systems, 14, 418-425.

Chen, J., \& Patton, R. J. (1999). Robust model-based fault diagnosis for dynamic systems. Boston: Kluwer Academic.

Chow, E., \& Willsky, A. (1984). Analytical redundancy and the design of robust failure detection systems. IEEE Transactions on Automatic Control, 29(7), 603-614.

De Persis, C., De Santis, R., \& Isidori, A. (2001). Nonlinear actuator fault detection and isolation for a VTOL aircraft. In Proceedings of the 2001 American control conference (pp. 4449-4454). Anchorage.

Edelmayer, A., Bokor, J., \& Szabo, Z. (2003). A geometric view on inversion-based detection filter design in nonlinear systems. In Proceedings of the fifth IFAC symposium on fault detection, supervision and safety of technical processes. SAFEPROCESS 2003 (pp. 783-788). Washington.

Eykeren, L. V., Chu, Q., \& Mulder, J. (2012). Sensor fault detection and isolation using adaptive extended Kalman filter. In Proceedings of the eighth IFAC symposium on fault detection, supervision and safety of technical processes. SAFEPROCESS 2012. Mexico City.
Frank, P. (1990). Fault diagnosis in dynamic systems using analytical and knowledge-based redundancy-A survey and some new results. Automatica, 26, 459-474.

Frank, P., Ding, S., \& Koppen-Seliger, B. (2000). Current developments in the theory of FDI. In A. Edelmayer (Ed.), Proceedings of the fourth IFAC symposium on fault detection, supervision and safety of technical processes. SAFEPROCESS 2000 (pp. 16-27). Budapest.

Goupil, P. (2010). Oscillatory failure case detection in the A380 electrical flight control system by analytical redundancy. Control Engineering Practice, 18, 1110-1119.

Goupil, P. (2011). Airbus state of the art and practices on FDI and FTC in flight control system. Control Engineering Practice, 19, 524-539.

Goupil, P., \& Marcos, A. (2012). Industrial benchmarking and evaluation of ADDSAFE FDD designs. In Eighth IFAC safeprocess, fault detection, supervision and safety of technical processes. Mexico City.

Hecker, S. (2006). Generation of low order LFT representations for robust control applications (Ph.D. thesis). Technische Universität München.

Khong, T. H., \& Shin, J. Y. (2007). Robustness analysis of integrated LPV-FDI filters and LTI-FTC system for a transport aircraft. In AIAA guidance, navigation, and control conference. Hilton Head, SC.

Ljung, L. (1999). System identification-Theory for the user (2nd ed.). Upper Saddle River, NJ: Prentice-Hall.

Marcos, A., Ganguli, S., \& Balas, G. (2005). An application of $\mathcal{H}_{\infty}$ fault detection and isolation to a transport aircraft. Control Engineering Practice, 13(1), 105-119.

Massoumnia, M. (1986). A geometric approach to the synthesis of failure detection filters. IEEE Transactions on Automatic Control, 31, 839-846.

Massoumnia, M., Verghese, G., \& Willsky, A. (1989). Failure detection and identification. IEEE Transactions on Automatic Control, 34, 316-321.

Patton, R. J., \& Chen, J. (1996). Robust fault detection and isolation FDI systems. Control Dynamic Systems, 74, 176-224.

Seiler, P., Vanek, B., Bokor, J., \& Balas, G. (2011). Robust $\mathcal{H}_{\infty}$ filter design using frequency gridding. In American control conference. San Francisco.

Spitzer, C. R. (Ed.). (2001). The Avionics handbook. Boca Raton, Florida: CRC Press.

Szaszi, I., Marcos, A., Balas, G., \& Bokor, J. (2005). LPV detection filter design for a Boeing 747-100/200 aircraft. AIAA Journal of Guidance, Dynamics and Control, 28(3), 461-470.

Tan, W. (1997). Applications of linear parameter-varying control theory (Master's thesis). University of California at Berkeley.

Van den Bossche, D. (2006). The A380 flight control electrohydrostatic actuators, achievements and lessons learnt. In Proceedings of the 25th congress of the international council of the aeronautical sciences. Hamburg.

Vanek, B., Seiler, P., Bokor, J., \& Balas, G. (2011). Robust fault detection filter design for commercial aircraft. In Euro GNC 2011 first CEAS specialist conference on guidance, navigation \& control. Münich.

Vanek, B., Szabó, Z., Edelmayer, A., \& Bokor, J. (2011). Geometric LPV fault detection filter design for commercial aircrafts. In AIAA guidance, navigation, and control conference. Portland.

Vanek, B., Szabó, Z., Edelmayer, A., \& Bokor, J. (2012). Fault detection of electrical flight control system actuators using parameter dependent estimation. In Proceedings of the eighth IFAC symposium on fault detection, supervision and safety of technical processes. SAFEPROCESS 2012. Mexico City.

Varga, A. (2003). On computing least order fault detectors using rational nullspace bases. In Proceedings of the fifth IFAC symposium on fault detection, supervision and safety of technical processes. SAFEPROCESS 2003. Washington. 